\title{
Erratum to: Curvilinear coordinate transformations for relative motion
}

\author{
David A. Vallado $^{1} \cdot$ Salvatore Alfano $^{1}$
}

Published online: 17 March 2016

(C) Springer Science+Business Media Dordrecht 2016

\section{Erratum to: Celest Mech Dyn Astr (2014) 118:253-271 DOI 10.1007/s10569-014-9531-1}

Pg. 258. The equation for $\hat{S}$ should be

$$
\hat{S}=\hat{W} \times \hat{R}
$$

Pg. 260. Equation 6 should not have an initial minus sign. The $\hat{P}$ term should have a minus sign, and the $\hat{Q}$ symbol should be within the parentheses.

$$
\vec{v}_{c h f_{P Q W 2}}=\sqrt{\frac{\mu}{p_{c h f}}}\left(-\sin \left(v_{2}\right) \hat{P}+\left(e_{c h f}+\cos \left(v_{2}\right)\right) \hat{Q}\right)
$$

Pg. 260. Equation 10 should have a cosine in the middle term denominator:

$$
\frac{\vec{v}_{d e p_{S E Z}}(e)}{\left|\vec{r}_{d e p_{R S W 1} \mid}\right| \cos \left(\Delta \phi_{d e p}\right)} r_{c h f 2}-\vec{v}_{c h f_{R S W 1}}(s)
$$

Pg. 262. Unnumbered equation before equation 15 should not have the arcsine in the first line.

$$
\Delta \phi_{d e p}=\left(\frac{z}{r_{c h f 2}}\right)
$$

The online version of the original article can be found under doi:10.1007/s10569-014-9531-1.

$凶$ David A. Vallado

dvallado@agi.com; davallado@gmail.com

Salvatore Alfano

salfano@agi.com

1 Center for Space Standards and Innovation, Analytical Graphics Inc., 7150 Campus Dr., Suite 260,

Colorado Springs, CO 80920-6522, USA 
Pg. 262 Unnumbered equation before equation 17.

- The first line should have a minus sign in the front and should use $r_{c h f 2}$ for consistency;

$$
-\frac{\dot{z}}{r_{c h f 2}} \frac{\vec{r}_{d e p_{S E Z}}(z)}{\hat{r}_{d e p_{S E Z}}(z)}
$$

- In the second line, the $r_{c h f 2}$ should be moved from within the first parenthesis term, to the last term;

$$
\left(\dot{y}+\vec{v}_{c h f_{R S W 1}}(s)\right) \frac{\vec{r}_{d e p_{S E Z}}(z)}{\hat{r}_{d e p_{S E Z}}(z)} \frac{\cos \left(\Delta \phi_{d e p}\right)}{r_{c h f} 2}
$$

